\title{
A Cell Biology Inspired Model for Managing Packet Broadcasts in Mobile Ad-hoc Networks
}

\author{
S.J. Sooriyaarachchi, W.A.C. Fernando and C.D. Gamage
}

\begin{abstract}
This Paper proposes a packet delivery mechanism for Mobile Ad hoc Networks (MANETs) in which the nodes are not required to keep global routing information and where there are no key nodes that act as infrastructure. The solution proposed in this Paper is based on blindrebroadcasting with packet storm control through a mechanism inspired by the chalone mechanism found in biological cell divisions. The operation of the proposed protocol is mathematically modelled using cellular automata. The efficiency of the proposed protocol was compared with those of blindrebroadcasting and sequence number controlled flooding using an OMNET simulation with respect to the total number of copies of the same data packet received in the network against the number of nodes in the network. The results show a significant improvement in the performance. The protocol efficiency was also analysed with respect to the spreading of the data packet against time using an analytical model based on an epidemic model. The results show comparable performance.
\end{abstract}

Keywords: Mobile ad-hoc networks, Packet broadcasts, Cellular automata.

\section{Introduction}

The modern day-to-day communication is moving towards an intensive use of mobile devices such as smart phones and slate/tab type computers that are capable of connecting via cellular networks, WiFi or Bluetooth. The ubiquity and portability of such devices give rise to a new computing experience such as converting a traditional classroom to a collaborative learning environment via a standalone ad hoc network of mobile phones. This Research focuses on a dense network of mobile devices that could be visualized as a dense Mobile Ad Hoc Network (dense MANET).

MANETs are dynamic in terms of topology, thus making it challenging to implement a routing mechanism. There is an added complexity into routing when these MANETs are dense in nature. For example the routing tables maintained in mobile nodes using the existing routing schemes such as AODV, TORA and DSR will become unmanageably large in size and the network will get overloaded with flooded control packets. Therefore, it is essential that the nodes in a dense MANET keep a minimum of state information or none at all, specifically those pertaining to the global network and even the neighbouring nodes. It is also important that the nodes send the least amount of control packets to conserve resources such as battery life. Due to the unavailability of a suitable routing mechanism the users have to rely on cellular networks without utilizing other communication technologies such as WiFi or Bluetooth even when the devices are at line of sight from each other.

Blind-rebroadcasting is the simplest form of packet routing that allows the nodes to be stateless. However, this scheme will cause broadcast storms, thus, resulting in severe degradation in performance, scalability and efficiency. Hence, the current research problem is to find a mechanism to send a data packet from one node to another in a dense MANET while minimizing broadcast storms and leaving the nodes to be stateless.

Biological inspirations have given promising results in arriving at satisfactory solutions for difficult problems similar to the above, especially in communication networks. For instance Wedde etal [1]inspired by the foraging principles of honey bees proposes an energy efficient routing algorithm for MANETs to address the energy dilemma in them. Gunes etal [2] have addressed the issue of packet overhead in routing and have proposed a routing algorithm inspired by swarm intelligence in ant colonies. There have been other studies based on ant colony optimizations

S.J.Sooriyaarachchi, B.Sc.Eng. (Peradeniya), M.Sc.(Moratuwa),Lecturer, Department of Computer Science and Engineering, University of Moratuwa. Dr. W.A.C. Fernando, B.Sc.Eng.(Moratwa), M.Sc.(AIT), PhD(Bristol), Reader, Centre for Vision, Speech and Signal Processing, University of Surrey, UK.

Eng. Lt. Col. (Dr.) C.D. Gamage, B.Sc. Eng. (Moratwa), M.Eng. (AIT), PhD (Monash), Senior Lecturer, Department of Computer Science and Engineering, University of Moratuwa. 
[3][4]. Moreover, Sarafijanovic and Boudec [5] address the problem of node misbehaviour in MANETs gaining inspiration from the human immune system for their solution. Similarly, Kefalas etal [6] have based their solution for the problem of modelling dynamic behaviour of multi-agent systems on the evolution of human tissues.

There are biological systems that show characteristics similar to those of a dense MANET and which try to achieve a common task by cooperating among autonomous entities. An organ in human body for instance is also made out of a dense population of biological cells. These cells divide themselves repetitively in a process called cell proliferation which is analogous to re-broadcasting of packets in a MANET. It is an interesting question to ask "On what basis does our ears end up growing into a particular size?" The nature has its regulatory mechanisms for controlling the cell divisions in an organ and this can inspire to find a mechanism to manage redundant rebroadcasts in a dense MANET.

\section{Related Work}

This Section briefs the routing approaches available in the area of MANETs and as to how the proposed approach differs from them.

\subsection{Traditional Routing}

Traditional routing approaches in MANETs are based on the routing protocols of wired networks. Data communication networks originated with the intention of connecting fixed wired terminals. The need for routing mechanisms arose as a result of the growth of the networks in size and with their geographical spreading. The networks started to support wireless nodes with the intension of using existing communication resources [7]. Hence, the wireless nodes always had the support of some kind of fixed infrastructure so that the wired routing protocols that existed at that time were tweaked to support a few wireless nodes. Dedicated fixed infrastructure such as base stations and wireless access points in local area networks were implemented in cellular networks when the mobile nodes increased in number [8]. However, the concepts behind almost all routing protocols used in MANETs are hooked to fixed infrastructure at least by assuming a few key nodes in the network to act as fixed and routing nodes. The most recent work in the area of MANETs is task group "s" amendment to the IEEE 802.11 of year 2012, which captures MANETs underWireless Mesh Networks. Mesh Stations in IEEE 802.11 standard act as Access Points in infrastructure mode wireless networks thus managing routing and control [9]. The routing paradigm that bases on fixed infrastructure and requires maintaining routes is considered as the fixed-stateful approach.

However, the current trend in MANETs seeks a paradigm shift as various mobile devices emerge in large numbers with powerful nearfield wireless networking capabilities. In parallel, the supporting technologies and concepts for ubiquitous computing and connectivity develop at a rapid phase. For example, IPv6 allows a large population of mobile devices to connect to a single network, while Bluetooth provides powerful near field communication. Hence it is preferred thatMANET routing mechanisms are based on the opposite of the fixed-stateful paradigm. The approach proposed in this Paper is based on the mobile-stateless approach.

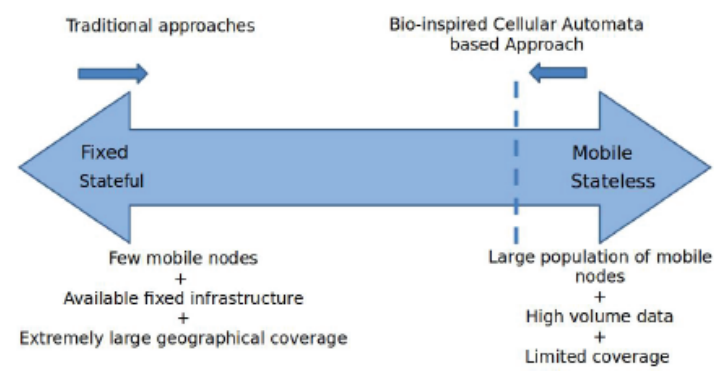

Figure 1 - Summary of the comparison of the proposed approach with reference to fixed stateful and mobile stateless routing paradigms

\subsection{Mobile-stateless Routing}

A comparison of the proposed approach and the traditional approaches of MANET routing is summarized in Figure 1. The simplest and stateless mechanism of sending a packet from one node to another in a MANET is blind rebroadcasting. In this mechanism a node rebroadcasts each and every packet it receives, except for those destined to the node itself. However, it causes an unnecessary amount of redundant rebroadcasts especially when the MANET is dense. Hence the solution proposed in this Paper is based on blind rebroadcast and it attempts to reduce redundant rebroadcasts using biologically inspired regulatory mechanisms. These regulations in turn require the maintenance of acertain amount of states thus shifting the proposed approach slightly 
towards stateful side as shown in Figure 1.

However, the states maintained in this approach do not capture global network information as in traditional routing approaches. The proposed approach requires information on itself and the immediate neighbourhood along with those carried by the received data packets.

Although there are routing protocols proposed based on cell biological inspirations, such as in [10] their solutions are also AODV-like protocols that maintain routing tables without considering node densities. Cellular Automata based models are also found as in [11] and [12] but they are either not in the scope of dense MANETs or they are not related to routing.

\subsection{Network-wide Reach in MANETs}

It is often essential that the packets are sent to every node in a MANET, at least for housekeeping tasks such as discovering routes and updating routing tables in traditional MANET routing. For example, AODV which is the most popular MANET routing protocol, relies on Sequence Number Controlled Flooding as the mechanism to send RREQ packets throughout the network [13]. IEEE 802.11 also uses a variation of AODV named HWMP, as the default routing (termed as path selection because it operates at link layer) protocol [14]. Hence, flooding is a decisive aspect of MANETs.

The initial idea for this work was intended to route data packets based on blindrebroadcasting. However, the findings are applicable in enhancing the available routing protocols which rely on some kind of flooding, to reach all nodes in a network. Hence this work is also a contribution to the improvement of flooding in MANETs.

\section{The Biological Model Based Approach}

This Section explains the biological inspirations for limiting the packet rebroadcasts in a MANET based on biological control mechanisms related to organ growth.

\subsection{Limiting the Packet Growth inside the MANET}

The aim was to control the growth of accumulated copies of the same data packet due to rebroadcasts inside a MANET with the inspiration gained from the organ growth control by inhibiting cell proliferation. Since a human organ is generally made out of millions of cells it is an ideal system to mimic the dense nature of the mobile network of interest in this Research. Any regulatory mechanism that controls cell proliferation in the growth of such organs would be a biological inspiration to manage broadcast storms in the target network.

According to Lui and Baron [15], the human body has a growth deceleration mechanism driven at the physiological level by growth itself and at a molecular level by a cell intrinsic genetic program. This claim is supported by Finkielstain et al [16]. Lui and Baron[15] further elaborate that the local communication mechanisms play a central role in growth deceleration rather than the systemic mechanism. Bryant and Simpson [17] also highlight the evidence for organ intrinsic mechanisms in growth control. However, the growth deceleration program is not cell autonomous and depends on the interaction of the cell with the extra-cellular micro environment [15].

\subsection{Using Growth as the Inhibitor of Growth}

The genetic program explained by Lui and Baron [15] is responsible for growth inhibition according to the inputs related to growth itself such as the number of accumulated cells, organ functionality and the number of cell divisions undergone. This genetic program up or downregulates the growth promoting genes according to a negative feedback loop. The hypothesis that the growth itself causes growth inhibition is also proved by Forcinito etal [18].

\subsection{Terminal Conditions of Growth}

According to Lui and Baron[15], the Growth Program described above down-regulates the growth-promoting genes when (i) the accumulated number of cells reach the saturation level, (ii) the organ is adequately grown to perform the intended functionalityor (iii) the number of divisions undergone is equal to a particular number set during the embryonic stage.

\subsection{Growth Sensing Mechanisms}

Individual cells sense the growth in terms of the number of accumulated cells by means of the concentration of some of the secreted molecules known as chalones [15]. Bullough [19] coined the term "chalone" and hypothesized it as the inhibitors of cell growth through negative feedback to control the size of a tissue. A number of studies provide experimental 
evidence in support of this hypothesis [20], [21],[22],[23].The growth in terms of the level of functionality is sensed for example, in the liver by the flux of bile acids. Bile acids are synthesized in the liver and secreted into the small intestine to facilitate digestion. Bile acid is then reabsorbed and returned to the liver. The number of cell divisions undergone is measured using cell cycle counting mechanisms such as telomere shortening [15].

\section{The Cell Biology Inspired Packet Transmission Model}

Work presented in this Paper, at this stage has been heavily inspired by the chalone method of growth inhibition in biological organ as explained in Section 3.4. This Section explains how the chalone mechanism is applied to limit packet rebroadcasting in MANETs.

The number of packets in a MANET grows by rebroadcasts analogous to the cells that proliferate by dividing themselves. Hence a data packet residing in a mobile node is mapped to a biological cell. The packet rebroadcasting is mapped to biological cell division. Table 1 summarizes the mapping from a biological system to MANETs.

The chalone concentration sensed by a cell in its micro-environment represents the number of cells in the neighbourhood in the biological system. Similarly the number of neighbours who have already broadcast a particular data packet is sensed using a chalone-like mechanism to decide whether a node should rebroadcast the data packet in hand. The term chaloneis borrowed for the control packets introduced in this Paper as cues for carrying the above information on the neighbourhood of MANETs. The control packet that inquires whether a neighbour has rebroadcast a specified data packet is named as chaloneInit to denote the initiation of the chalone mechanism. The control packet sent by neighbours in response to chaloneInit is named Chalone packet. These control packets are never forwarded beyond the transmission range of a node.

Table 1: Summary of Mapping from Biological Sys-
tem to MANET
\begin{tabular}{|l|l|}
\hline $\begin{array}{l}\text { Biological } \\
\text { System }\end{array}$ & MANET \\
\hline $\begin{array}{l}\text { Biological } \\
\text { cell }\end{array}$ & Data packet in a node \\
\hline Cell division & Packet rebroadcasting \\
\hline Chalone & $\begin{array}{l}\text { A control packet exchanged only with } \\
\text { the neighbors. Neighbors are the } \\
\text { nodes within the transmission range } \\
\text { of a node. }\end{array}$ \\
\hline $\begin{array}{l}\text { Chalone } \\
\text { concentra- } \\
\text { tion }\end{array}$ & $\begin{array}{l}\text { Number of neighboring nodes who } \\
\text { have already broadcasted the Data } \\
\text { Packet. }\end{array}$ \\
\hline
\end{tabular}

\section{Operational Model based on Cellular Automata}

Typically the functional behaviour of cell biological systems is modelled in terms of Cellular Automata [24], [25], [26], [27]. Similarly, the operation of a biologically inspired broadcasting model for MANETs is theoretically analysed in this Section based on Cellular Automata.

Cellular Automata (CA) is defined as the mathematical idealization of problems or physical systems in which space and time are discrete and the physical values take on a finite set of discrete values [28]. This was invented by John von Neumann in 1940s as a result of biological motivation, with the intention of designing self-replicating artificial systems. Power of CA lies in its fundamental properties that are also found in the physical world: CA is massively parallel, homogeneous and all interactions are local [29]. Therefore a variety of physical and biological systems have successfully been modelled and simulated using CA [25], [30], [31],[32],[33].

One-dimensional two-state CA is extensively elaborated in [28]. The formalism of CA is found in [34] for multi-dimensions which is an exhaustive algebraic treatment of the subject. The type of CA that is of interest in this Research is two-dimensional as the nodes in the MANET lie effectively on a plane. Packard and Wolfram[35] further analyse two-dimensional $\mathrm{CA}$ and cites [34] for additive two-dimensional CA. A two-dimensional CA consists of a regular lattice of sites [35]. Each site takes on $\mathrm{k}$ number of possible values. Each site is updated in discrete time steps according to a set of rules, $\varnothing$ that depends on the values of sites in some neighbourhood around it at the previous time step. Packard and Wolfram[35] address the special class of $\varnothing$ known as totalistic rules in which the values of a site depends only on the sum of values of the neighbours.

This CA model of MANET is also viewed as a regular lattice on which mobile nodes are randomly placed. Each site takes only two values: 1 - node has rebroadcast a specified data packet, 0 - otherwise. Neighbourhood for the CA in this Research is the circular region captured by the radio range of a mobile node. Any node within the range will respond as a neighbour. Here also the totalistic rule is applied where the total number of neighbours 
who have rebroadcast a specified data packetis counted.

\section{Analytical Model}

Khelil [36] proposed an epidemic model to analyze message spreading in broadcasts considering the similarity between message spreading among MANET nodes and spreading of infectious diseases among living beings. This is a generalized analytical model which is used to evaluate broadcasting mechanisms considered in this Research.

The SI epidemic model in epidemiology is used for developing the above analytical model. In this model, an individual is mapped to one of the two compartments, namely, Susceptible (S) and Infectious (I). Once infected, an individual remains infected forever and keeps on infecting others until the whole population gets infected.

The SI model for packet broadcasting, defines a variable named spreading ratio, $i(t)$ that denotes the ratio between the number of nodes that received the message to the total number of nodes. Simulation results related to $i(t)$ is used in this Paper as a measure of the protocol efficiency with respect to time.

\section{Simulation}

The proposed protocol and the two related protocols were simulated in OMNET++ (version 4.4) with INET (version 2.2.0) which is an event-driven and modular simulation framework [37]. The simulation model under study comprised of an array of hosts configured as nodes of a single private network and its important parameters are listed in Table 2.

Table 2: Simulation parameters

\begin{tabular}{|l|l|}
\hline Parameter & Value \\
\hline playground size & $600 \mathrm{~m} \times 400 \mathrm{~m}$ \\
\hline bitrate & $54 \mathrm{Mbps}$ \\
\hline transmitter range & $200 \mathrm{~m}$ \\
\hline chalone timeout & $0.1 \mu \mathrm{s}$ \\
\hline mac header length for data & 20 Bytes \\
\hline $\begin{array}{l}\text { mac header length for chalone } \\
\text { messages }\end{array}$ & 6 Bytes \\
\hline
\end{tabular}

As in well-known flooding algorithms, a data packet is uniquely identified here by its source address and a sequence number which in combination is referred to as Packet ID hereafter. The sequence number is assigned to a packet by the originator of that packet. A node maintains a list of Packet IDs it sees or it creates. It also keeps a list of neighbours who respond for a chaloneInit message. When a node receives a data packet, a flow of events occurs as indicated in Figure 2. It is assumed that chalone packets are quite small compared to data packets (as given in table 2) because they need to carry only the MAC address of the sender.

On the receipt of a chaloneInit message, the source node ignores it while the destination is supposed to initiate another type of negative feedback mechanism similar to mechanism (ii) given in Section 3.2.However, this feedback mechanism does not come under the scope of this Paper. Flooding is for the global broadcast in a MANET using typical routing mechanisms and the terminal condition is when a packet reaches all the nodes at least once. For unicast the terminal condition is reached when the packet reaches its intended destination. The negative feedback initiated by destination therefore is supposed to reduce the redundant rebroadcasts upon reaching the terminal condition at unicast in a MANET. Nodes other than the source and the destination that receive a chaloneInit would respond with a Chalone message. Chalone message carries the neighbour state defined in Section 5.

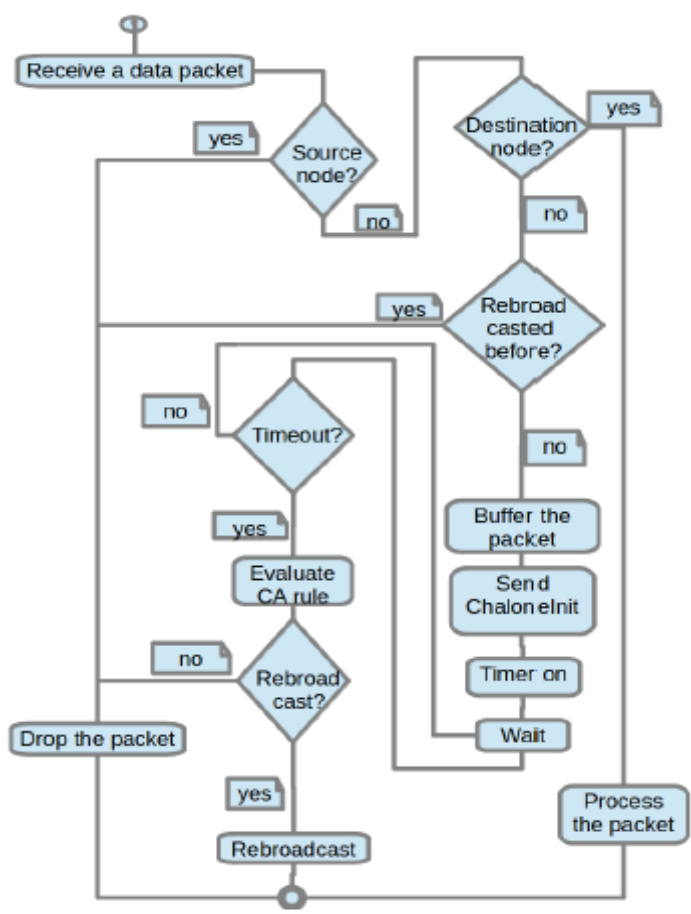

Figure 2 - The flow of events upon the receipt of a data packet by a node

In this Paper, the case of unicast is investigated by specifying a source node and a destination for a data packet, Thus the success of a 
transmission is indicated when the packet has reached the destination. Blind-rebroadcasting, sequence number controlled flooding and Cellular Automata based rebroadcasting are compared in Section 8.

\section{Results and Discussion}

This Section presents the analysis of the efficiency of the proposed protocol (CA) against blind-rebroadcasting (blind) and sequence number controlled flooding (SNCF) with respect to two performance metrics: (i) the number of times a particular data packet is received by any node in the MANET until the destination is reached and (ii) spreading ratio (explained in Section 6) over time until the destination is reached. Metric (i) is averaged over 10 simulation runs and is shown in Figure 3 with a 99\% confidence level. Nodes were placed randomly in these simulation runs according to 10 different seeds given to the default random number generator in OMNET++.

According to Figure 3, the CA protocol outperforms both blind and SNCF protocols for all node densities. For example in a MANET with 100 nodes, blind-rebroadcast caused the network to receive the same packet around 2440 times on average. The worst case scenario that can be assumed in a 100 node network is where the packet reaches its destination after going through all the other 98 nodes. Thereby it should record only 98 copies of the same packet in the network for the best possible protocol. In contrast, blind protocol has a waste of over 2000 packets according to the results obtained. The SNCF protocol recorded about 1870 times whereas the CA protocol reported only around 1000 times. Thus the CA protocol saves nearly $60 \%$ of the redundant rebroadcasts compared to blind-rebroadcasting whereas there is a saving of nearly $45 \%$ compared to SNCF.

Also for a node density of 1000 , the counts were: more than 280,000 packets for blindrebroadcasting, around 210,000 for SNCF and only around 70,000 packets for CA protocol. Blind-rebroadcasts are unacceptable for dense MANETs in terms of redundant rebroadcasts as the growth of the curve is steeply exponential. Though SNCF is slightly better compared to blind-rebroadcasting, it also shows an exponential growth. CA on the other hand shows a slow growing curve thus making it a better protocol for all node densities shown. The results show satisfactory performance and usability of the proposed protocol in dense MANETs as the number of redundant rebroadcasts is almost linear and there is a slow growth against node density. CA protocol in small MANETs of 20 nodes also shows a saving of redundant rebroadcasts of around $40 \%$ and $10 \%$ compared to blind and SNCF protocols respectively.

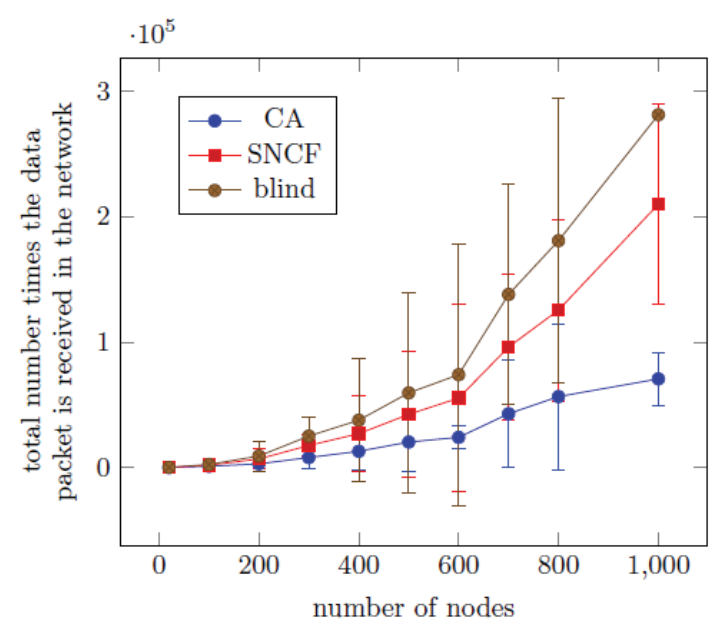

Figure 3 - Number of times the same data packet is received until the destination is reached for blind, SNCF and CA protocols

Run number 3 was analysed for protocol efficiency with respect to time at different node densities and the relevant plots of spreading ratio are given in Figure 4. This particular run number was observed to give packet counts closer to the averages considered in Figure 3 and avoided special cases such as when the destination is in the immediate neighbourhood of the source node.

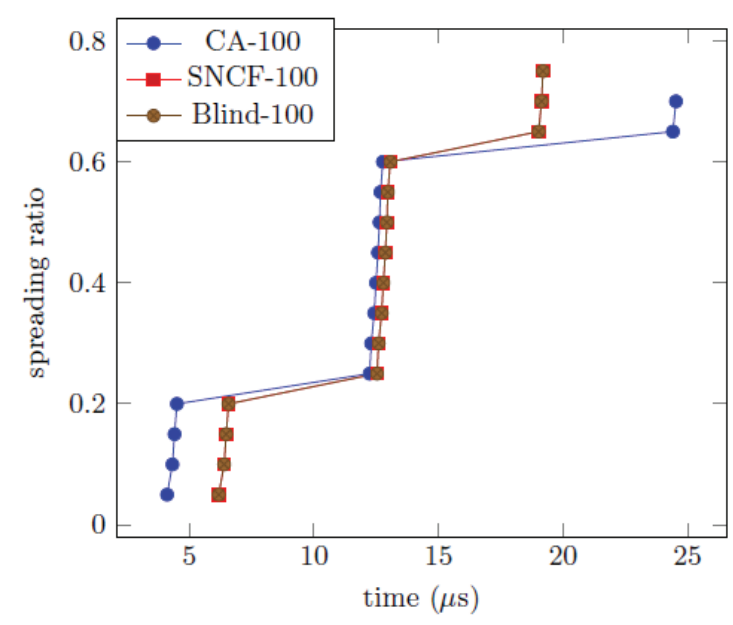

Figure 4 - Spreading ratio vs time until the destination is reached in a 100-node network for blind, SNCF and CA protocols. Other node densities gave similar results. 
According to Figure 4, the time performance among the CA, blind and SNCF protocols shows no significant difference though the CA protocol starts the process a few microsecond earlier and finishes a little later. However, this time difference may also be omitted by possible optimizations as to how the state information should be kept and by obtaining the required neighbour information via received packets and physical layer cues instead of special chalone messages.

\section{Conclusion}

Network-wide packet broadcasting is an essential aspect of any routing protocol including HWMP which is the default Wireless Mesh routing (path selection) protocol given in the latest IEEE 802.11 standard. However HWMP also uses flooding as the mechanism to broadcast its control packets throughout the network. It was found through simulations that flooding as well as blind-rebroadcasting donot perform satisfactorily in MANETs when the node densities becomehigher. In contrast, a Cellular Automata based broadcast mechanism was proposed in this Paper inspired by biological cell proliferation in human organs. The chalone mechanism from organ growth process was borrowed into MANET rebroadcasting and a limited broadcast scenario was proposed which gave a saving of over $10 \%$ of redundant rebroadcasts compared to both sequence number controlled flooding and blind-rebroadcasting for low node densities and over $45 \%$ of savings for higher node densities above 100 nodes without compromising performance with respect to time.

In the next phase of this Research Project, the bio-inspired CA based MANET routing approach presented in this Paper will also be tested with different mobility models applicable in practical dense MANETs. Further optimization of the protocol state through an analysis of received packets and use of physical layer cues instead of special chalone messages to avoid collisions will also be tested.

\section{References}

1. Wedde, H. F., Farooq, M., Pannenbaecker, T., Vogel, B., Mueller, C., Meth, J., \& Jeruschkat, R., "BeeAdHoc: An Energy Efficient Routing Algorithm for Mobile Ad Hoc Networks Inspired by Bee Behavior", Proc. Genetic and Evolutionary Computation Conference, pp. 153160, June, 2005.
2. G"unes, M., Sorges, U.,\& Bouazizi, I., “ARA The Ant-Colony Based Routing Algorithm for MANETs",Proc. IWAHN 2002,pp. 79-85, August, 2002.

3. Schoonderwoerd, R., Bruten, J. L., Holland, O. E.,\& Rothkrantz, L. J. M., “Ant-Based Load Balancing in Telecommunications Networks", J. Adapt. Behav.,Vol. 05, No. 02, pp. 169-195, 1996.

4. Caro, G. D., Ducatelle, F., \& Gambardella, L. M., "AntHocNet: an adaptive nature-inspired algorithm for routing in mobile ad hoc networks", J. European Transactions on Telecommunications, Vol. 16, No. 05, pp. 443455, September, 2005.

5. Jean-yves, \& Boudec L., “An Artificial Immune System for Misbehavior Detection in Mobile Ad-Hoc Networks with Virtual Thymus, Clustering, Danger Signal and Memory Detectors", J. Artificial Immune Systems, pp. 342-356, 2004.

6. Kefalas, P., Eleftherakis, G., Holcombe, M., \& Stamatopoulou, I., "Formal Modelling of the Dynamic Behaviour of Biology-Inspired, Agent-Based Systems“, Molecular Computational Models: Unconventional Approaches", 2005, pp.243-244.

7. Abramson, N., "Development of the ALOHANET", J. IEEE Transactions On Information Theory, Vol. IT-31, No. 02, pp. 119123, March, 1985.

8. Dubendorf, V. A., Wireless Data Technologies, John Wiley and Sons Ltd, 2003, ch. 1.

9. Hiertz, G. R., Denteneer, D., Max, S., Taori, R., Cardona, J., Berlemann, L., \& Walke, B., "IEEE 802.11s: The Wlan Mesh Standard", J. IEEE Wireless Communications", Vol. 17, No. 01, pp. 104-111, February, 2010.

10. Liu, Z., Kwiatkowska, M. Z., \& Constantinou, C., "A Biologically Inspired QoS Routing Algorithm for Mobile Ad Hoc Networks", J. IJWMC, Vol. 04, No. 02, pp. 64-75, May, 2010.

11. Mullany, F. J., Ho, L. T. W., Samuel, L. G., and Claussen, H., "Self-deployment, Selfconfiguration: Critical Future Paradigms for Wireless Access Networks", Autonomic Communication, International Federation for Information Processing, 2004, pp.58-68.

12. Subrata, R., \& Zomaya, A. Y., "Evolving Cellular Automata for Location Management in Mobile Computing Networks", J. IEEE Transactions on Parallel and Distributed Systems, Vol. 14, No. 01, pp. 13-26, January, 2003. 
13. http://www.ietf.org/rfc/rfc3561.txt, Visited, 10th Dec 2014.

14. Conner, W. S., Kruys, J., Kim, K., \& Zuniga, J. C., "IEEE 802.11s Tutorial: Overview of the Amendment for Wireless Local Area Mesh Networking", IEEE 802 Plenary, November,2006.

15. Lui, J. C., \& Baron, J., "Mechanisms Limiting Body Growth in Mammals", J. Endocrine Reviews, Vol. 32, No. 03, pp. 422-440, June, 2011.

16. Finkielstain, G. P., Forcinito, P., Lui, J. C., Barnes, K. M., Marino, R., Makaroun, \& Baron, J., "An Extensive Genetic Program Occurring during Postnatal Growth in Multiple Tissues", J. Endocrinology, Vol. 150, No. 04, pp. 1791-1800, April, 2009.

17. Bryant, P. J., \& Simpson, P., "Intrinsic and Extrinsic Control of Growth in Developing Organs", J. The Quarterly Review of Biology, Vol. 59, No. 04, pp. 387-415, December, 1984.

18. Forcinito, P., Andrade, A. C., Finkielstain, G. P., Baron, J., Nilsson, O., \& Lui, J. C., "Growth-inhibiting Conditions Slow Growth Plate Senescence", J. Endocrinol, Vol. 208, No. 01, pp. 59-67, January, 2011.

19. Bullough, W. S., "Mitotic and Functional Homeostasis:A Speculative Review", J. Cancer Research, Vol. 25, No. 10, pp. 1683-1727, November, 1965.

20. Onda, H., "A New Hypothesis on Mitotic Control Mechanism in Eukaryotic cells", J. Theoretical Biology, Vol. 77, No. 03, pp. 367377, April, 1979.

21. Lee, S. J., "Regulation of Muscle Mass by Myostatin", J. Annual Review of Cell and Developmental Biology, Vol. 20, pp. 61-86, 2004.

22. Elgjo, K.,\& Reichelt, K. L., “Chalones: from Aqueous Extracts to Oligopeptides", J. Cell Cycle, Vol. 03, No. 09, pp. 1208-1211, September, 2004.

23. Bakthavatsalam, D., Brock, D. A., Nikravan, N. N., Houston, K. D., Hatton, R. D., \& Gomer, R. H., "The secreted Dictyostelium protein CfaD is a chalone", J. Cell Science, Vol. 121, pp. 2473-2480, August, 2008.

24. Kansal, A. R., Torquato, S., Harsh, G. R., Chiocca, E. A.,\& Deisboeck, T. S., “Simulated Brain Tumor Growth Dynamics Using a Three-Dimensional Cellular Automaton",J. Journal of Theoretical Biology, Vol. 203, No. 04, pp. 367-382, April, 2000.
25. Lee, Y., Kouvroukoglou, S., McIntire, L. V., \& Zygourakis, K., “A Cellular Automaton Model for the Proliferation of Migrating Contact-inhibited Cells", J. Biophysical Journal, Vol. 69, pp. 1284-1298, October, 1995.

26. Alarcóna, T., Byrneb, H. M., \& Mainia, P. K., "A Cellular Automaton Model for Tumour Growth in Inhomogeneous Environment", J. Theoretical Biology, Vol. 225, No. 02, pp. 257274, November, 2003.

27. Mireira, O., \& Deutsch, A., "Cellular Automaton Models of Tumor Development: A Critical Review", J. Advs. Complex Syst, Vol. 05, No. 2/3, pp. 247-268, 2002.

28. Wolfram, S., "Statistical Mechanics of Cellular Automata", J. Reviews of Modern Physics, Vol. 55, No. 03, pp. 601-644, July, 1983.

29. Kari, J., "Theory of Cellular Automata: A Survey", J. Theoretical Computer Science, Vol. 334, pp. 3-33, November, 2005.

30. Barredo, J. I., Kasanko, M., McCormick, N., \&Lavalle, C., "Modelling Dynamic Spatial Processes: Simulation of Urban Future Scenarios through Cellular Automata", J. Landscape and Urban Planning, Vol. 64, pp. 145160, 2003.

31. Liu, Q.X., Jin, Z., \& Liu, M.X., "Spatial Organization and Evolutional Period of the Epidemic Model using Cellular Automata", J. Physical Review E,Vol. 74, November,2006.

32. Li , X. B., Jiang, R., \& Wu, Q. S., “Cellular Automaton Model Simulating Traffic Flow atan Uncontrolled T-Shaped Intersection", J. International Journal of Modern Physics B, Vol. 18, No. 17-19, pp. 2703-2707, 2004.

33. Almeida, R. M., \& Macau, E. E. N., “Stochastic Cellular Automata Model for Wildland Fire Spread Dynamics", Proc. 9th Brazilian Conference DINCON'10,pp. 249-253, June,2010.

34. Martin, O., Odlyzko, A. M., \& Wolfram, S., "Algebraic Properties of Cellular Automata", J. Communications in Mathematical Physics, Vol. 93, pp. 219-258, 1984.

35. Packard, N. H., \& Wolfram, S., "TwoDimensional Cellular Automata", J. Journal of Statistical Physics, Vol. 38, No. 5/6, pp. 901946, 1985.

36. http://elib.uni-stuttgart.de/opus/volltexte/ 2007/3120/pdf/khelil_diss.pdf, Visited, 10th Nov 2014.

37. http://www.omnetpp.org/doc/omnetpp/ manual/usman.html, Visited, 10th Dec 2014. 